ORIGINAL ARTICLE

\title{
Evaluation of the status of tuberculosis as part of the clinical case definition of AIDS in India
}

\author{
V S S Attili, V P Singh, M Rai, D V Varma, S Sundar
}

Postgrad Med J 2005;81:404-408. doi: 10.1136/pgmj.2004.025726

See end of article for authors' affiliations

.....................

Correspondence to: Dr V S S Attili, Room 104, PG Hostel, Kidwai Memorial Institute of Oncology, Dr Mari Gowda Road, Bangalore 560029, India; sureshattili@yahoo.com

Submitted 17 June 2004 Accepted

28 September 2004

\begin{abstract}
Aim: To assess HIV associated tuberculosis in a high tuberculosis prevalence setting and its status in the clinical case definition of AIDS.

Methods: All HIV patients attending the infectious disease clinic, Varanasi, India between January 2001 and December 2003 were included in the study. They were stratified into three distinct immunological categories depending on their CD4 levels in accordance to Centers for Disease Control (CDC) classification. Tuberculosis of different organs was defined as detailed below.

Results: Tuberculosis was the commonest opportunistic disease, seen in 163 patients. Of these, 68 had exclusively pulmonary tuberculosis, 55 extrapulmonary disease, and 40 the disseminated form. Pulmonary and extrapulmonary tuberculosis had low positive predictive value (PPV) (51\% and 42\%) for CD4 levels of $<200$ when compared with the disseminated form (specificity $87 \%$ and PPV $75 \%$ ). Among 86 patients with radiological evidence of tuberculosis, typical radiological features of post-primary tuberculosis were present in 60 cases (70\%). Other features such as effusion (14 patients, 16\%) and miliary shadows (12 patients, 14\%) were comparatively rare.

Conclusion: Keeping pulmonary and extrapulmonary forms of tuberculosis in AIDS defining illness should be reconsidered. In a similar way tuberculosis in HIV patients from areas endemic with tuberculosis occurs in patients with a wide range of immune status and has a better prognosis than other AIDS defining illnesses. Therefore the inclusion of tuberculosis in clinical case definition of AIDS is not justified.
\end{abstract}

$\mathrm{H}$ IV was identified in 1983 and was shown to be the aetiological agent of AIDS in 1984. With the availability of sensitive and specific diagnostic tests for HIV infection, and for the immune status of the patient, the case definition of AIDS has undergone several revisions. The definition of AIDS was established not for the practical care of the patients but for the surveillance purposes. The Centers for Disease Control and World Health Organisation classifications were the most widely used ones. However, different countries have developed and adopted their own classification systems. In India the National AIDS Control Organisation definition is used for surveillance purposes. It had long been debated to keep the diseases with high prevalence in the clinical case definition of AIDS, which point to a fairly advanced HIV disease. In countries with low prevalence of tuberculosis (TB), it can serve the purpose of representing as an "opportunistic infection". The role of TB as and opportunistic infection in countries like South Africa was questioned by Badri et $a l^{5}$ after prospective cohort studies. Therefore we conducted a study to find the pattern of TB in HIV patients and its status in the clinical case definition of AIDS. We also evaluated other clinical diseases for their predictive value of low CD4 counts.

\section{METHODS}

\section{Patients}

All HIV infected patients attending the infectious diseases (ID) clinic, SS hospital, Varanasi between January 2001 and December 2003 were included in the study. From the total 450 patients who attended the clinic, CD4 counts were available in 375 patients (the remainder either did not consent or data were missing). Informed consent was obtained from all the patents and all the examination findings and investigations were recorded in the ID records. The patients were stratified into three distinct immunological categories deepening on their CD4 levels. CD4 of $<200 / \mathrm{mm}^{3}$ was taken as severe immunosuppression, CD4 of $>500 / \mathrm{mm}^{3}$ was taken as near normal immune status, and a value in between these was taken as intermediate immune suppression.

\section{CD4 cell estimation}

Immunophenotyping of lymphocytes was carried out by FACS count (Becton Dickinson, Singapore (BD)) lymphocytes were stained according to the protocol suggested by the manufacturer. CD4 count was calculated using the formula as suggested in the manual.

\section{HIV serology}

HIV status of the patients was confirmed by enzyme linked immunosorbent assay using two different antigens.

\section{Definitions of TB \\ Pulmonary TB}

Diagnosis was confirmed by positive sputum examination for acid fast bacillus (AFB) or characteristic findings on chest radiograph, or both.

\section{Extrapulmonary TB}

Diagnosis of tubercular meningitis was based on history of subacute or chronic meningitis, cerebrospinal fluid showing increased protein levels, lymphocytic pleocytosis along with raised adenosine deaminase activities above 7 IU/l with the finding of AFB. In cases with no AFB, patients who responded with therapeutic trial of antitubercular therapy were included as tubercular meningitis. In a similar way abdominal tuberculosis was confirmed by either ascetic fluid examination or suggestive barium studies or by

Abbreviations: TB, tuberculosis; AFB, acid fast bacillus; PPV, positive predictive value 


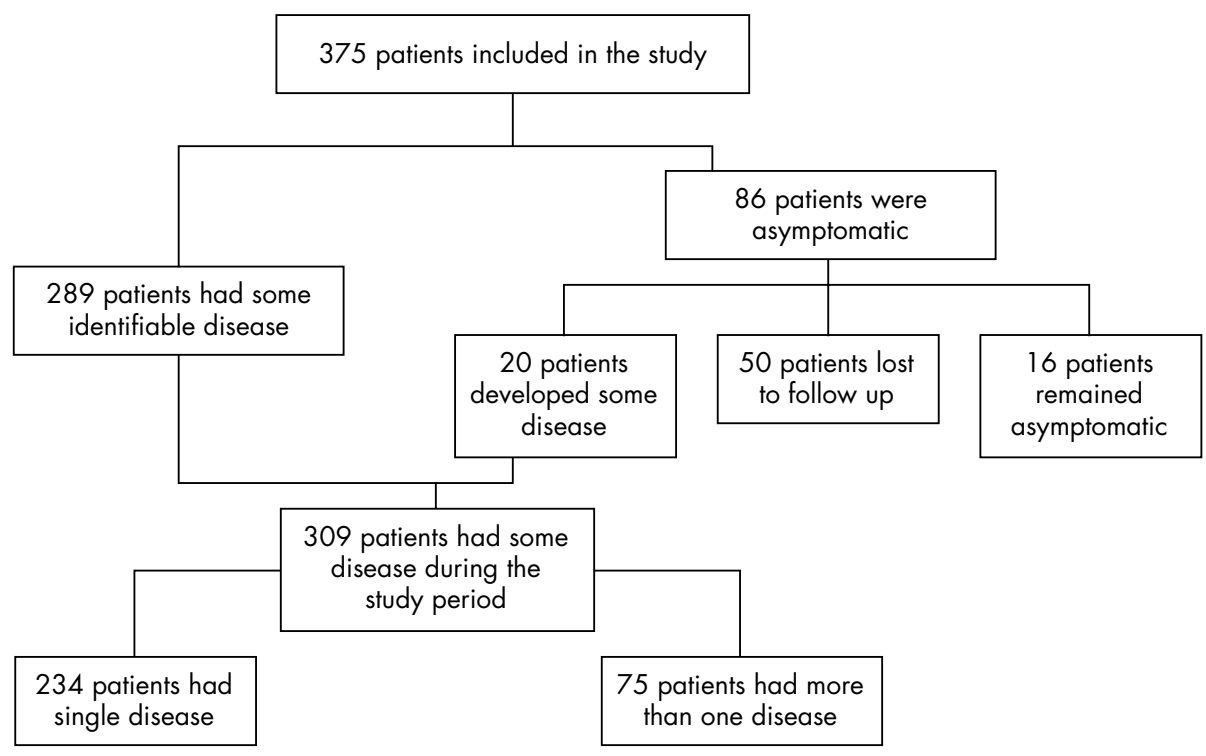

Figure 1 Follow up of the patients.

ultrasonographic guided fine needle aspiration cytology from abdominal lymph nodes.

\section{Lymph node TB}

Diagnosed by the finding of caseation on biopsy or finding of AFB by fine needle aspiration cytology.

\section{Disseminated TB}

Diagnosed by involvement of two or more organ systems by TB.

\section{Sample collection and microscopy}

In all patients with productive sputum, samples were collected in wide mouth containers. In those patients with dry cough, sputum was induced by nebulising the patient with normal saline. The samples were stained with the ZeihlNielsen stain. In a similar way cerebrospinal fluid, ascetic fluid, and plural fluid were also examined.

\section{Statistical analysis}

The main characterisation of the association between CD4 counts below 200 and different clinical and radiological presentations was the positive predictive value (PPV). This was compared between different presentation types using the Kruskal-Wallis test on actual CD4 levels, in preference to a less powerful $\chi^{2}$ test. Sensitivity and specificity are not appropriate measures to compare between the three mutually exclusive and exhaustive TB classifications listed in table 1 , because sensitivities automatically total $100 \%$, as do complements of specificities. Nevertheless it was judged appropriate to show specificity as well as positive predictive value for disseminated TB in table 3, as comparators for the other presentation types shown.

\section{RESULTS}

Figure 1 shows the follow up of the patients.

We recruited all patients presenting during 2001-3. We present here cross sectional results based on findings at

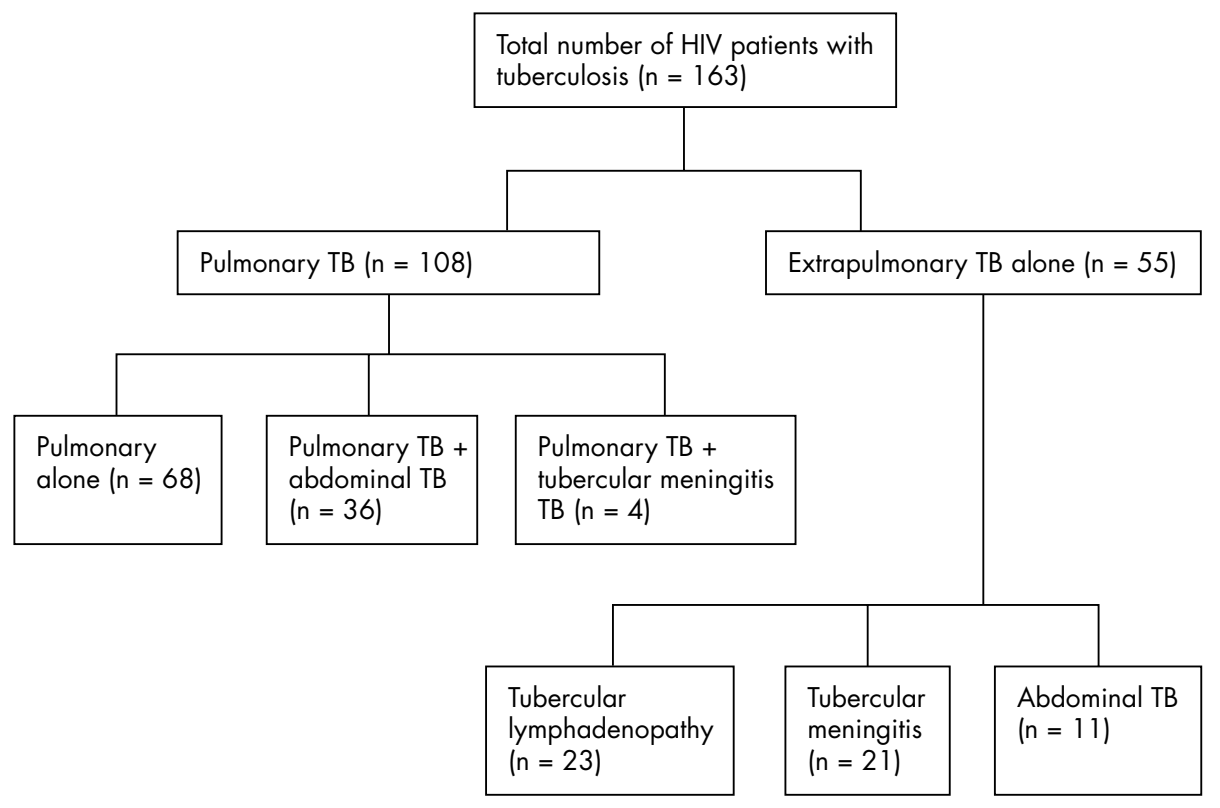

Figure 2 Distribution of TB cases. 


\begin{tabular}{|c|c|c|c|c|}
\hline Type of tuberculosis & $\begin{array}{l}\text { CD4 }<200 \\
(n=88)\end{array}$ & $\begin{array}{l}\text { CD4 200-500 } \\
(n=58)\end{array}$ & $\begin{array}{l}\text { CD4 }>500 \\
(n=17)\end{array}$ & $\begin{array}{l}\text { Total } \\
(n=163)\end{array}$ \\
\hline Pulmonary alone & $35(51)$ & $28(41)$ & $5(8)$ & 68 \\
\hline Extrapulmonary & $23(42)$ & $22(40)$ & $10(18)$ & 55 \\
\hline Tubercular lymphadenopathy & $2(9)$ & $13(56)$ & $8(35)$ & 23 \\
\hline Tubercular meningitis (TBM) & $13(62)$ & $7(33)$ & $1(5)$ & 21 \\
\hline Abdominal tuberculosis & $8(73)$ & $2(18)$ & $1(9)$ & 11 \\
\hline Disseminated tuberculosis & $30(75)$ & $8(20)$ & $2(5)$ & 40 \\
\hline Pulmonary TB + TBM & $4(100)$ & $0(0)$ & $0(0)$ & 4 \\
\hline Pulmonary $\mathrm{TB}+$ organomegaly & $26(72)$ & $8(22)$ & $2(1)$ & 36 \\
\hline
\end{tabular}

presentation. Follow up was planned, but is incomplete and is unlikely to yield useful information because of poor follow up.

Pulmonary tuberculosis was suspected in 108 patients and 86 of these patients had evidence of tuberculosis by chest radiography (findings include cavity in 22 cases, upper zone infiltrates in 38 cases, miliary shadows in 12 cases, and pleural effusion in 14 cases). In 22 patients, there was a strong clinical suspicion of tuberculosis and AFB in sputum was positive despite normal chest radiograph. In 36 patients there was associated organomegaly and these were diagnosed as disseminated tuberculosis. Eleven patients had hepatosplenomegaly with mesenteric lymphadenopathy or thickened bowel loops. These were included in the category of exclusive abdominal tuberculosis. Surprisingly none of the patients with tubercular lymphadenopathy had active tuberculosis by radiography .

The overall range of TB was pulmonary 68 , disseminated 40 , and extrapulmonary 55 . Table 1 shows the details of the immune status of TB patients according to the various forms of TB. The results show that there are pronounced differences in the immune status of the HIV patients who also had various forms of $\mathrm{TB}$. As the sensitivities and specificities would inevitably total to 1 , because of dependence on the calculation based on the same sample, we applied the Kruskal-Wallis test to compare the actual CD4 counts between the three groups. The results suggested that the difference was significant $(p<0.0001)$ in the three forms of the TB.

We correlated the radiological findings to the CD4 (table 2). By looking at the gross values it might seem that cavitary lesions and miliary shadows had better predictive value compared with the other lesions for low CD4. But statistical analysis showed that none of the radiological findings were good enough for the low CD4 prediction $(\mathrm{p}>0.05)$.

Table 3 shows the results of the evaluation of the specificity and PPV of different clinical conditions in predicting the CD4 levels. Most of the CDC category $\mathrm{C}$ diseases (cryptococcal meningitis, progressive multifocal leucoencephalopathy, pneumocystis carinii pneumonia, CNS oxoplasmosis, recurrent herpes zoster, and disseminated molluscum contagiosum) had a specificity of more than $90 \%$ in predicting the low CD4 counts. Although disseminated TB had figures reaching this value none of the other forms of TB reached the optimal figure. The 95\% confidence intervals of the CD4 levels in most of these diseases also were around 200 (apart from oral candidiasis, toxoplasmosis, and CCM, and in the last two the difference is attributable to the small number of patients).

As it is known that with decreasing immunity the number of opportunistic infections will increase, we evaluated the CD4 levels of the HIV patients with TB when they had other opportunistic infections. If we compare the predictive value of TB when it was associated with other diseases (for example, oral candidiasis, recurrent herpes zoster, etc), the results showed that its specificity improved. So it might be considered an opportunistic infection when associated with other diseases (table 4).

\section{DISCUSSION}

Tuberculosis in its different forms is the commonest reported disease in HIV patients in different series published from India. $^{2-4} 9^{10} 13^{14} 4_{16-21}$ It is also the commonest AIDS defining illness reported by NACO. ${ }^{16}$ The pattern and contribution of TB in our study was similar to others. ${ }^{6-8} 1112$ We also analysed the sputum positivity, radiological pattern, risk factors, and effect of TB and immune status on survival.

\section{Sputum positivity}

The sputum positivity in HIV patients ranged from $15.4 \%$ to $85 \%$ depending on the immune status of the patients in different series. ${ }^{16}{ }^{22}$ Chances of AFB isolation were high in patients with mild immunosuppression compared with the advanced disease in the above mentioned studies. In this study, the sputum positivity was $44.2 \%$ (comparable to other studies and indirectly implicating comparatively good immunity).

\section{Radiological pattern}

Radiological lesions reported by various other authors include pulmonary infiltrates $(7 \%-56.3 \%)$, cavity $(25 \%-33 \%)$, miliary

Table 2 Immunological status of 86 patients with radiological evidence of tuberculosis according to radiological findings

\begin{tabular}{lllll}
\hline Type of lesion & $\begin{array}{l}\text { CD4 }<200 \\
(\mathbf{n}=58)\end{array}$ & $\begin{array}{l}\text { CD4 200-500 } \\
(\mathbf{n}=20)\end{array}$ & $\begin{array}{l}\text { CD4 }>500 \\
(\mathbf{n}=8)\end{array}$ & $\begin{array}{l}\text { Total } \\
(\mathbf{n}=86)\end{array}$ \\
\hline $\begin{array}{l}\text { Upper zone infiltrates } \\
\text { (bilateral) }\end{array}$ & $24(63)$ & $8(21)$ & $6(16)$ & 38 \\
Cavity & $18(82)$ & $3(14)$ & $1(4)$ & 22 \\
Effusion & $7(50)$ & $7(50)$ & $0(0)$ & 14 \\
Miliary & $9(75)$ & $2(17)$ & $1(8)$ & 12 \\
\hline
\end{tabular}

Percentages shown in parentheses. Kruskall-Wallis test $p=0.1201$. 
Table 3 Specificity and positive predictive value of different diseases in predicting the CD4 levels of $<200 / \mathrm{mm}^{3}$

\begin{tabular}{lcccc}
\hline Disease & Number of cases & Specificity $\left.{ }^{*} \%\right)$ & PPV* (\%) $^{*}$ & Mean*(CD4) (SD) \\
\hline Disseminated tuberculosis & 40 & 87 & 75 & $163(111)$ \\
CNS toxoplasmosis & 5 & 99 & 80 & $145(70)$ \\
PML & 3 & 100 & 100 & $127(18)$ \\
CCM & 16 & 98 & 81 & $186(104)$ \\
PCP & 16 & 97 & 71 & $147(32)$ \\
Candidiasis & 81 & 69 & 38 & $237(141)$ \\
Recurrent herpes zoster & 12 & 97 & 66 & $138(105)$ \\
Molluscum contagiosum & 6 & 99 & 83 & $145(37)$ \\
\hline
\end{tabular}

PCP, Pneumocystis carinii pneumonia; CCM, cryptococcal meningitis; PML, progressive multifocal leucoencephalopathy. *All the values were rounded to the nearest whole number.

shadows $(6.3 \%-16.7 \%)$, and diffuse infiltrates (12.5\%$13 \%),{ }^{13} 131721$ (none of them correlated it with immune status). We found typical radiological features of postprimary $\mathrm{TB}$ - that is, upper zone infiltrates in 38 patients $(44 \%)$ and cavitary lesions in 22 patients $(26 \%)$. Other features like effusion in 14 patients (16\%), miliary shadows in 12 patients $(14 \%)$ were rarely seen. Our findings suggested that cavitary lesions and miliary shadows had better predictive value compared with the other lesions. The pattern of pulmonary involvement and the frequency of extrapulmonary involvement in this study were not different from other Indian reports.

\section{Risk factors for TB}

Various studies exist globally, evaluating the risk factors for development of TB (most of them conducted in countries with low TB prevalence). In most of them neither age, sex, nor the mode of transmission of HIV behaved as significant risk factors for development of TB and the only observed risk factor was the country of origin and patients from areas of high prevalence had higher chances of developing TB..$^{15}$

\section{Effect of TB and immune status on the survival}

We evaluated the effect of TB and immune status on the survival by analysing various studies. In a study conducted by Stonburner et al, the authors found that HIV patients with TB had higher mortality compared with those who did not and suggested that pulmonary $\mathrm{TB}$ be included in the clinical case definition of AIDS. ${ }^{24}$

Studies by Perronne $\mathrm{C}$ et al in France, also had similar results (note, the difference in CD4 levels of pulmonary and extrapulmonary forms of $\mathrm{TB}$ were not different in that study). ${ }^{25}$

However, a few years after these two small studies, a large prospective study conducted over 17 years on 1000 AIDS cases at Puerrto Roico, found that patients with TB alone had higher survival compared with those with other AIDS defining illnesses. They also found that patients with TB and AIDS with lower CD4 levels had higher mortality

Table 4 Specificity and positive predictive value of TB when it is associated with other diseases (for example, oral candidiasis, recurrent herpes zoster, molluscum contagiosum)

\begin{tabular}{lcl}
\hline Disease & $\begin{array}{l}\text { Specificity* } \\
(\%)\end{array}$ & $\begin{array}{l}\text { Positive predictive } \\
\text { value* (\%) }\end{array}$ \\
\hline Oral candidiasis & 96 & 88 \\
Recurrent herpes zoster & 100 & 90 \\
Molluscum contagiosum & 100 & 94 \\
\hline *All the values were rounded to the nearest whole number.
\end{tabular}

compared with those with higher CD4 levels. This suggests that immunosuppression is the main reason for the death rate rather than the mere presence of $\mathrm{TB}^{26}$

In a similar way, a larger cohort of 18062 US HIV patients was analysed for validating 1993 revised clinical case definition of AIDS by CDC. The authors concluded that "AIDS-defining conditions first occurred in HIV-infected patients with CD4+ T-lymphocyte counts below $0.20 \times 10(9) / \mathrm{L}$ (200/microL) for $80 \%$ of diagnoses. Similarly, AIDS-defining diseases occurred at counts below $0.05 \times 10(9) / \mathrm{L}$ for $50 \%$ of diagnoses. Exceptions to both criteria were invasive cervical cancer and pulmonary tuberculosis". ${ }^{27}$

Studies from the developing countries were largely small hospital based studies. Initially Badri et al found that the onset of TB in HIV infected patients is associated with an increased risk of AIDS and death. Although a causal link was not established as it was an observational study, authors had the view that prolonged immune activation induced by TB increased HIV replication and consequent accelerated disease progression.

But the same author found a different result when he conducted a prospective cohort study. There he found that $67 \%$ of the TB cases occurred at a CD4 level much higher than $200 / \mathrm{mm}^{3}$. And the survival of patients with TB was comparable to that of other benign illnesses like oral candideasis. They also found that survival of patients with pulmonary and extrapulmonary TB was not different. ${ }^{5}$

In view of the considerable variability of the results across the globe, it is advisable to analyse the studies according to the prevalence of the individual diseases in respective countries.

In India TB is highly prevalent even in non-HIV patients, consequently it probably affects HIV patients in any stage of the disease. In this study TB affected 163 (43\%) patients. Of these patients, 124 patients had TB as the only AIDS defining illness. The CD4 count in the patients was quite variable, ranging from 3 to 610 .

We also evaluated the specificity of $\mathrm{TB}$ in predicting the low CD4 levels and the results suggested that pulmonary and extrapulmonary TB had low specificity (56\% and $57 \%$ ) and low PPV (51\% and $42 \%$ ) for CD4 levels of $<200 / \mathrm{mm}^{3}$ when compared with the disseminated form, which had a specificity of $87 \%$ and positive PPV of $75 \%$. So we conclude that keeping pulmonary and extrapulmonary forms (especially lymph nodal as it is the factor responsible for low positive predictive value) of $\mathrm{TB}$ in AIDS defining illness should be reconsidered.

TB in HIV patients from areas endemic with TB occurs in patients with a wide range of immune status and has a better prognosis than other AIDS defining illnesses. Therefore the inclusion of TB in clinical case definition of AIDS is not justified. Results of this study also support the views of Badri et al from South Africa who found that most of the TB affected patients (67\%) had CD4 level of more than $200 / \mathrm{mm}^{3}$. 
Limitations of the study:

- Small sample size

- Non-evaluation of the survival data

- Large attrition in the sample

- This study did not investigate the natural history of TB and HIV in India

Explanations/ suggestions:

- As the study was conducted in the Uttar Pradesh state of the India, which contained only 870 AIDS patients in January 2003, a larger study involving all states is required to look for the exact pattern

- As most of the asymptomatic HIV patients do not seek medical attention, and patients might have TB for some time it is difficult to predict the natural history of $\mathrm{TB}$ in HIV patients.

\section{CONCLUSION}

Retaining pulmonary and extrapulmonary forms (especially lymph nodal as it is the factor responsible for low PPV) of TB in AIDS defining illness should be reconsidered. In a similar way TB in HIV patients from areas endemic with TB occurs in patients with a wide range of immune status and has a better prognosis than other AIDS defining illnesses. Therefore the inclusion of TB in the clinical case definition of AIDS is not justified.

\section{ACKNOWLEDGEMENTS}

We thank Mr Robert Newcombe for help with the statistical analysis.

\section{Authors' affiliations}

V S S Attili, V P Singh, M Rai, D V Varma, S Sundar, Institute of Medical Sciences, Banaras Hindu University, Varanasi, India

Funding: none.

Conflicts of interest: none declared.

\section{REFERENCES}

1 Abouya L, Coulibla IM, Coulibaly D, ef al. Radiological manifestations of pulmonary tuberculosis in HIV-1 and HIV-2 infected patients in Abidjan, Cote $\mathrm{d}^{\prime}$ Ivoire. Tuber Lung Dis 1995;76:436-40.

2 Agrawal SK, Makhija A, Anuradha S, et al. The spectrum of opportunistic infections in HIV/AIDS patients in a tertiary care hospital in New Delhi. XIV International AIDS conference, Barcelona, 7-12 Jul, 2002:No.PeB-7220.

3 Arora VK, Kumar SV. Pattern of opportunistic pulmonary infections in HIV sero-positive subjects: observations from Pondicherry, India. Indian J Chest Dis Allied Sci 1999:41:135-44.
4 Chakrabarty T, Banerjee KA, Battacharya M, et al. Spectrum of opportunistic infections in AIDS related complex among high risk groups in Calcutta, India 10 years study. International conference on AIDS 1999: No PB1241.

5 Badri M, Ehrlich R, Pulerwitz T, et al. Tuberculosis should not be considered an AIDS-defining illness in areas with a high tuberculosis prevalence. Int J Tuberc Lung Dis 2002;6:231-7.

6 Coleubinders R, Mann JM, Francis $\mathrm{H}$, et al. Evaluation of a clinical case definition of AIDS Africa. Lancet 1987;i:472-4.

7 Elliott AM, Luo N, Tembo G. Impact of tuberculosis in Zambia: a crosssectional study. BMJ 1990;301:412-15.

8 George J, Hamide A, Das AK, et al. Clinical and laboratory profile of 60 patients with AIDS: a South Indian study. Southeast Asian J Trop Med Public Health 1996;27:686-91

9 Kaur A, Babu PG, Jacob M, et al. Clinical and laboratory profile of AIDS in India. J Acquir Immune Defic Syndr 1992;5:883-9.

10 Kothari K, Goyal S. Clinical profile of AIDS. J Assoc Physicians India 49:435-9.

11 Kumar $\mathbf{P}$, Sharma N, Sharma NC, et al. Clinical profile of tuberculosis patients with HIV infection/AIDS. Indian J Chest Dis Allied Sci 2002;41:159-63.

12 Kumarasamy N, Solomon S, Jayaker Paul SA, et al. Spectrum of opportunistic infections among AIDS patients in Tamil Nadu, South India. Int J STD AIDS 1995;6:447-9.

13 Mohanty KC, Sundrani RM, Nair S. HIV seropositivity in patients with respiratory disease. Indian J Tuber 1993;40:5-12.

14 NACO. NACO report on AIDS in India, Dec 2001. http://www.naco.org.

15 Pitkin $A D$, Grant AD, Foley NM, et al. Changing patterns of respiratory disease in HIV positive patients in a referral centre in the United Kingdom between 1986 and 7 and 1990-1. Thorax 1993:48:204-7.

16 Purohit SD, Gupta RC, Bhatura VK. Pulmonary tuberculosis and human immunodeficiency virus infection in Ajmer. Lung India 1996;14:113-20.

17 Rajasekaran S, Uma A, Kamakshi S, et al. Trend of HIV infection in patients with tuberculosis in rural South India. Indian J Tuber 2000:47:223-6.

18 Shankarnarayan S, Gagate A, Samani PH. Analytical study of clinical case profile of opportunistic infections in people living with HIV/AIDS in Mumbai, XIV international AIDS conference, Barcelona, 7-12 Jul 2002: No PeB-8215.

19 Sunderam G, McDonald RJ, Maniatis T, et al. Tuberculosis as a manifestation of the acquired immunodeficiency syndrome (AIDS). JAMA 1986;256:362-6.

20 Surana A, Vajpayee N, Seth P. Spectrum of opportunistic infections among HIV infected north Indian patients. XIV international AIDS conference, Barcelona, 7-12 Jul 2002: No PeB-7188.

21 Thanasekaran V, Krishnarajasekhar OR, Madhvi S, et al. Pulmonary disease in HIV infected patients S, et al. Pulmonary disease in HIV infected patients; an analysis of 16 cases. Lung India 1994;12:123-8.

22 Watson JM, Gill on. HIV infection and tuberculosis. BMJ 1990;300:63-5.

23 Sudre P, Hirschel BJ, Gatell JM, et al. Tuberculosis among European patients with the acquired immune deficiency syndrome. The AIDS in Europe Study Group. Tuber Lung Dis 1996;77:322-8.

24 Stoneburner $R$, Laroche $E$, Prevots $R$, et al. Survival in a cohort of human immunodeficiency virus-infected tuberculosis patients in New York City. Implications for the expansion of the AIDS case definition. Arch Intern Med 1992;152:2033-7.

25 Perronne C, Ghoubontni A, Leport C, et al. Should pulmonary tuberculosis be an AIDS-defining diagnosis in patients infected with HIV? Tuber Lung Dis 1992;73:39-44.

26 Perez-Perdomo R, Perez-Cardona CM, Suarez-Perez E. The epidemiology of tuberculosis in patients with AIDS in Puerto Rico: morbidity and survival, 1981-1998. Int J Tuberc Lung Dis 2000;4:713-18.

27 Hanson DL, Chu SY, Farizo KM, et al. Distribution of CD4+ T lymphocytes at diagnosis of acquired immunodeficiency syndrome-defining and other human immunodeficiency virus-related illnesses. The Adult and Adolescent Spectrum of HIV Disease Project Group. Arch Intern Med 1995;155:1537-42. 